\title{
Staged bronchial closure in uniportal video-assisted thoracoscopic anatomical resection for lung cancer with calcified lymph nodes
}

\author{
Ying-Yuan Chen, Tzu-Hung Lin, Chao-Chun Chang, Wei-Li Huang, Yi-Ting Yen, Yau-Lin Tseng \\ Division of Thoracic Surgery, Department of Surgery, National Cheng Kung University Hospital, College of Medicine, National Cheng Kung \\ University, Tainan, Taiwan \\ Correspondence to: Yau-Lin Tseng, MD, PhD. Department of Surgery, National Cheng Kung University Hospital, No.138, Shengli Rd., North Dist., \\ Tainan, Taiwan 704. Email: tsengyl@mail.ncku.edu.tw.
}

\begin{abstract}
Uniportal video-assisted thoracoscopic surgery (VATS) makes a breakthrough in these years. Even we have gained more experience and surgical skills of uniportal VATS, some elements, such as calcified perivascular lymph nodes, make the surgery challenging. In this series, we used staged bronchial closure (cut the bronchus first and then close it with stapler after dividing the pulmonary artery with calcified lymph node) as an approach for dealing with this challenging issue. Though the rate of intraoperative vessel injury is relatively high, we obtained ideal surgical outcome by using this technique in different lobes and segment of the lung.
\end{abstract}

Keywords: Uniportal; video-assisted thoracoscopic surgery (VATS); calcified lymph node; staged bronchial closure

Received: 26 July 2017; Accepted: 14 August 2017; Published: 28 October 2017.

doi: 10.21037 /jovs.2017.08.16

View this article at: http://dx.doi.org/10.21037/jovs.2017.08.16

\section{Introduction}

The first case of uniportal video-assisted thoracoscopic surgery (VATS) lobectomy was reported by Dr. Diego Gonzalez-Rivas in 2011 (1). Since then, the uniportal VATS lobectomy has become increasing popular for its better cosmetic result, less postoperative pain and shorter postoperative pleural drainage and hospital stay (2). Although the indication of uniportal VATS keeps extending, the compromised dexterity and safety is still a great concern comparing with multiportal VATS (3). Regarding to the technique difficulties in VATS, periarterial or peri-bronchial calcified lymph node remains one of the major causes of unplanned conversion (4). Herein, we present our experience about staged bronchial closure in dealing with calcified peri-arterial lymph node to achieve a successful uniportal VATS lobectomy and segmentectomy for lung cancer.

\section{Methods}

\section{Patient selection}

There were 309 patients undergoing uniportal VATS anatomical resection in the National Cheng Kung University Hospital from March 2015 to June 2017. We focused on the surgery using staged bronchial closure technique in managing calcified lymph node in lung cancer surgery. The data extracted included baseline demographics, preoperative evaluation, operative data, perioperative events, duration of pleural drainage, and postoperative hospital stay. This study was approved by the Institutional Review Board of the National Cheng Kung University Hospital.

\section{Preoperative preparation}

General anesthesia with selective one-lung ventilation was 


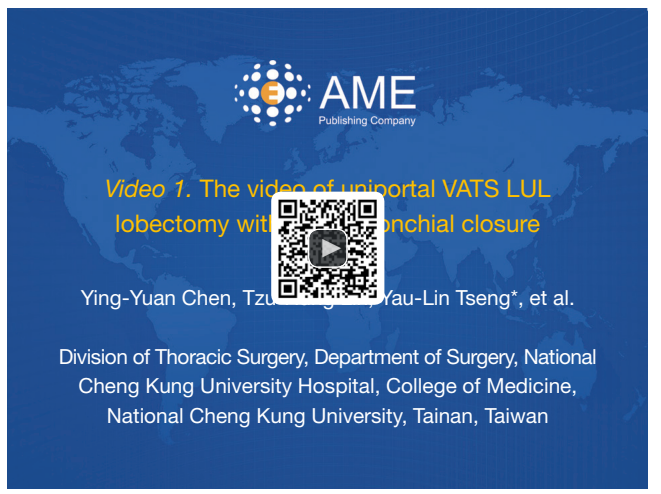

Figure 1 The video of uniportal VATS LUL lobectomy with stage bronchial closure (5). VATS, video-assisted thoracoscopic surgery; LUL, left upper lobe.

Available online: http://www.asvide.com/articles/1764

conducted through double-lumen or endotracheal tube or standard single-lumen endotracheal tube with bronchial blocker as anesthesiologist's decision. The patients were positioned in the full lateral decubitus position with both arms in pray posture. The operator always stood at the ventral site of the patient.

\section{Surgical technique}

A 3- to 4-cm incision was created in the 4th or 5 th intercostal space between the anterior and mid-axillary line as a surgeons' decision. An XS-sized Alexis wound protector (Applied Medical, Rancho Santa Margarita, CA, USA) was applied and $30^{\circ} 10-\mathrm{mm}$ thoracoscope was used for vision.

Our favorite instruments for dissection and retraction were hook electrocautery, D'Amico mediastinal biopsy forceps, Foerster lung grasping clamps, long right angle hemostatic forceps, and Wolf suction.

Lobectomy and segmentectomy were defined as division of lobar and segmental pulmonary artery and vein, division of lobar and segmental bronchus and resection of parenchyma. The pulmonary vessels were ligated by staples, surgical tie, or clips. The bronchus was closed by stapler device in all patients. There were 2 kinds of thoracoscopic stapler used in our hospital: Endo-GIA (Covidien, Mansfield, MA, USA) and Endo-cutter (Ethicon EndoSurgery, Guaynabo, Puerto Rico, USA). Stapling method was used to carry out segmentectomy and fused fissure to minimize air-leak from lung parenchyma.

Specimen was placed in self-sterilized plastic zipper storage bag and pulled out. Mediastinal lymph node dissection, including at least three stations of N2 nodes, was carried out as well. One chest tube was placed for pleural drainage through the wound after operation. The chest tube would be removed based on the drainage amount and status of air-leakage of lung.

\section{Staged bronchial closure technique}

When encountering a calcified periarterial lymph node, and for looping the segmental pulmonary artery safely, all other unaffected segmental pulmonary artery and pulmonary vein would be ligated or divided first. After dividing the pulmonary vein, which was rarely affected by calcified node, and removing peribronchial lymphatic tissue, the target bronchus usually could be identified. We used a long curved Metzenbaum scissors to cut the bronchus as distal as possible near the resected lobe or segment from the opposite site of the calcified node (Figure 1). It is important to keep a continuous suction through the endotracheal tube at the opened bronchus to prevent accumulation of blood clots in the airway postoperatively. After transecting the bronchus, we kept to remove the calcified node from the bronchial wall with a sharp dissection. Part of the calcified node would be left on the arterial wall. After gaining a sufficient length of proximal lobar of segmental bronchus, we used a linear stapler to close the opened bronchial stump. Finally, a vascular stapler could be used to remove the last segmental pulmonary artery with calcified node en bloc.

Sometimes, the bronchial wall may be torn or incised during sharply dissecting of the calcified node. A 3-0 or 4-0 Prolene suture wound be used to repair the torn bronchial wall prior to closing it (Figure 2). An air-leak test was conducted to make sure the safety of bronchial stump after all the procedures.

\section{Results}

There were eight patients undergoing uniportal VATS anatomical resection and mediastinal lymph node dissection for lung cancer by using staged bronchial closure technique between March 2015 and June 2017 (Table 1). There were 1 lingulectomy and 7 lobectomies performed in 5 left upper lobe, 1 right upper lobe and 1 right middle lobe. The mean operation time was 223 minutes (11-260 minutes) and the mean blood loss was $319 \mathrm{~mL}(0-900 \mathrm{~mL})$. Two patients underwent the salvage surgery after a treatment for their lung cancer including one with target therapy (case 1) and the other 


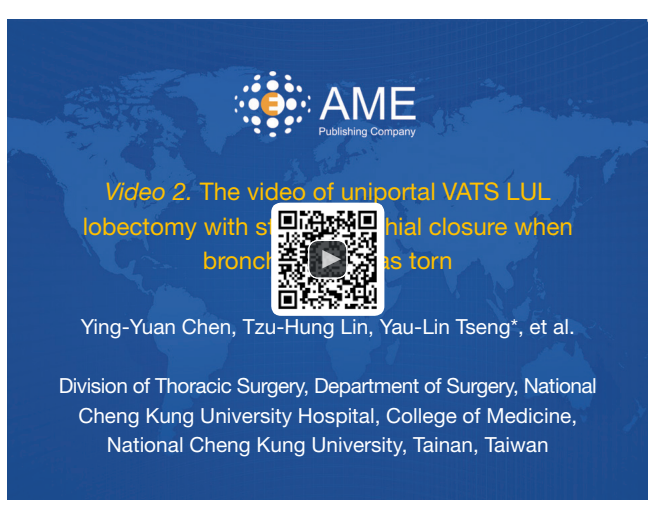

Figure 2 The video of uniportal VATS LUL lobectomy with stage bronchial closure when bronchial wall was torn (6).VATS, videoassisted thoracoscopic surgery; LUL, left upper lobe.

Available online: http://www.asvide.com/articles/1765

with chemotherapy (case 2). Intraoperative vascular injury, both pulmonary vein and pulmonary artery, during dissection was encountered in 5 of the 8 patients $(62.5 \%)$. All vascular injuries were controlled by suture repair without enlargement of the utility port or conversion to multiportal approach or open approach. The most common postoperative complication was prolonged air-leak $(25 \%, \mathrm{n}=2)$. The median duration of pleural drainage and postoperative hospital stay were 2.5 days (1-14 days) and 5.5 days (3-15 days), respectively.

\section{Discussion}

We presented a case series of primary lung cancers with calcified per-arterial lymph node that were managed successfully with staged bronchial closure technique under uniportal VATS lobectomy and segmentectomy.

Minimally invasive operation is a trend nowadays. The evolution of surgical approach of thoracic surgery starts from thoracotomy to multiportal thoracoscopy and then to uniportal thoracoscopy. For the decreasing wound size and operative posts, it is easily questioned whether minimal invasive surgery is safe and feasible. For example, in surgery for malignancy disease, surgeons may pay attention to the safe resection margin, radical lymph nodes clearance and long-term survival. This issue comes into uniportal surgery as well as debate between conventional open surgery and VATS surgery. It is not so clear yet since the development of uniportal surgery is making the move. However, in some studies and even meta-analysis study had proved the safety and the feasibility for uniportal lobectomy in there shortterm and intermediate follow $(2,7)$.
There were some conditions being regarded as technique difficulties in the developing era of multiportal VATS lobectomy, such as large tumors, centrally located tumors, severe pleural adhesion, fused fissure, previous chemotherapy or chemoradiation, or previous surgery, and lymph node calcinosis (4). Nearly all the technique difficulties could have been overcome by increasing experience in VATS surgery. However, the perivascular and peribronchial calcified lymph node remains one of the major cause of vascular injury and unplanned conversion of VATS lobectomy, which accounted for $36-41 \%$ of unplanned conversion $(8,9)$. Among them, calcifications around the bronchus of resection lobe had the highest risk of conversion (9). Therefore, how to manage the periarterial and peribronchial calcified lymph node is a really challenge issue especially in uniportal VATS.

Complete removal of regional lymph nodes is an important procedure in lung cancer surgery, not only for accurate staging but a definite treatment. Although metastasis rarely occurred in a calcified lymph node, there were still some case reports describing the possibility (10). Therefore, we do not divide the bronchus, pulmonary artery as well as the calcified lymph node simultaneously with a single stapler cartridge. The staple may be broken and part of lymph node will be retained on the stump. In our method, the lymph node can be removed completely with the pulmonary artery. Even if the calcified node is hard to be separated from the bronchus, the bronchus could be partially resected and repaired by suture before sealed by stapler without loss of stump integrity (Figure 2).

Wang and colleague had reported similar technique in dealing with a left-upper-lobe lung cancer with a calcified lymph node adhesive tenaciously to the bifurcation of lingular and basilar pulmonary artery under uniportal VATS approach (11). In this study, we applied the staged bronchial closure technique not only to left upper lobe, but to right upper lobe, right middle lobe and lingular segment. Among the cases of left upper lobe lobectomy, the most common site of calcified node was between first three branches of pulmonary artery and left upper lobe bronchus. Stage bronchial closure technique could avoid the disastrous vascular injury to the first three branches of left pulmonary artery.

In our experience, staged bronchial closure can also be used in left upper lobe lobectomy with fused interlobar fissure. After dividing the pulmonary artery to apical and anterior segment and superior pulmonary vein, we can divide the bronchus first with stapler first if the upper lobe bronchus can be looped with right angle hemostats 


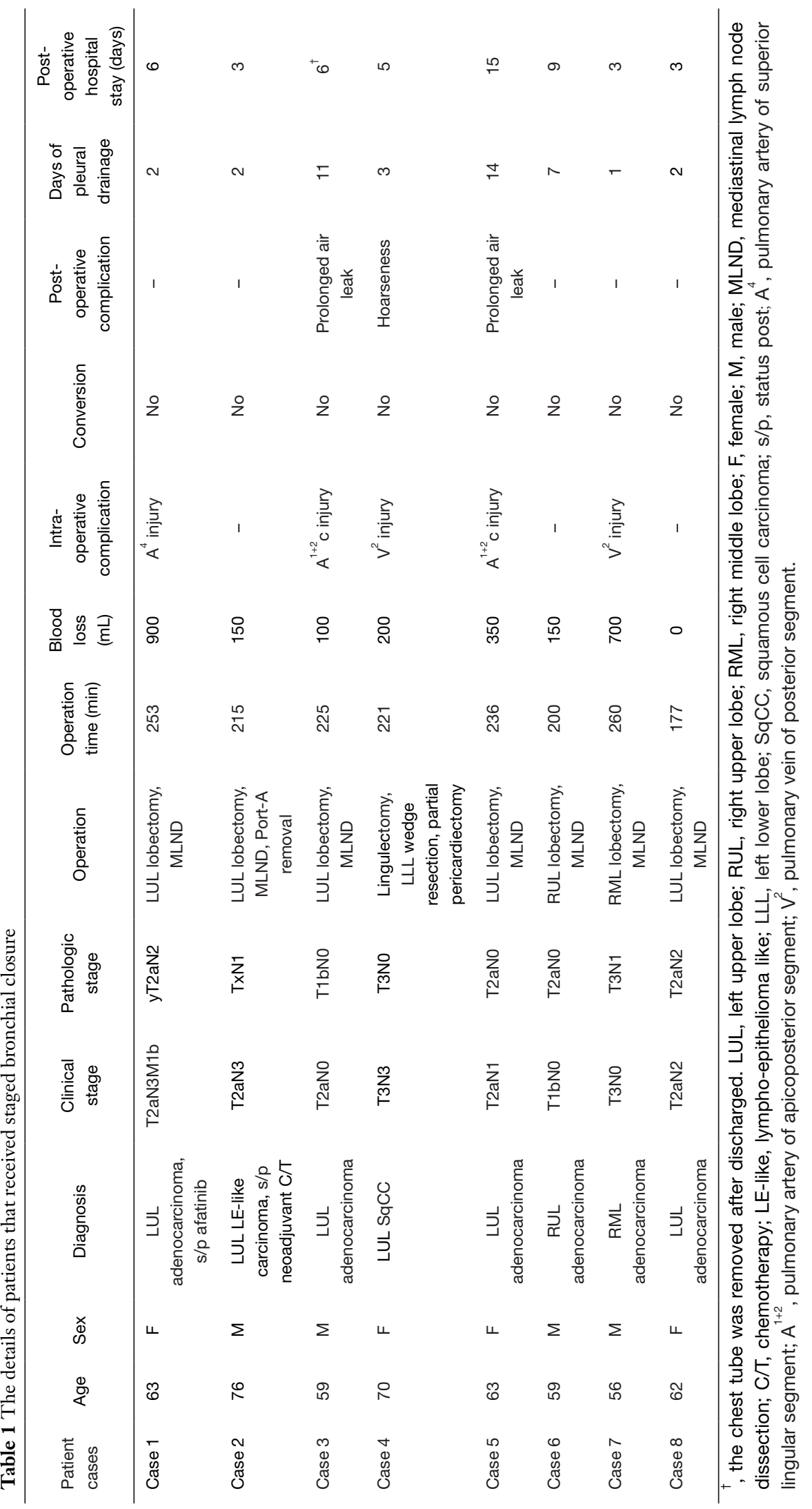


easily. The pulmonary arteries to posterior and lingular segment are visible and approachable at this time. Then, the fused interlobar fissure is divided thoroughly by staplers to minimize the occurrence of air-leak. Once the left upper lobe bronchus cannot be looped easily. This occurs in the situation such as the space behind bronchus is not wide enough or the lip of stapler will conflict with the pulmonary artery or lung parenchyma. Then the staged bronchial closure technique can facilitate the following procedures and make the operation safe and smoothly. We had ever done one case by using this technique.

The rate of vascular injury was high in our series (5 in 8 cases). This happened frequently because of the dense adhesion between the calcified nodes and pulmonary artery. Actually, all the vascular injuries in this series were minor and could be controlled with uniportal suture repair by this technique; any form of conversion was not needed. Moreover, it is much easier to repair a vascular injury from the front side rather than from the back side of vessels.

This study has some limitation. It is a singleinstitutional experience and the case number is still small. Also, most of the cases performed by this technique were in left upper lobe lobectomy. We ever applied this technique in lower lobe lobectomy by multiple ports VATS. To our thought, this method may be limited in uniportal VATS lobectomy in bilateral lower lobe. Because in our approach from anterolateral aspect of the 4th or 5th intercostal space, the bronchus of lower lobe is behind the pulmonary artery and is difficult to approach by Metzenbaum scissors theoretically. We will attempt the feasibility of this technique in lower lobe lobectomy in the future by uniportal VATS.

In conclusion, uniportal VATS lobectomy with staged bronchial closure is feasible in management of lung cancer with calcified periarterial lymph nodes around resected bronchus, especially in bilateral upper lobe, right middle lobe and left lingular segment.

\section{Acknowledgements}

None.

\section{Footnote}

Conflicts of Interest: The authors have no conflicts of interest to declare.

\section{References}

1. Gonzalez-Rivas D, de la Torre M, Fernandez R, et al. Single-port video-assisted thoracoscopic left upper lobectomy. Interact Cardiovasc Thorac Surg 2011;13:539-41.

2. Harris CG, James RS, Tian DH, et al. Systematic review and meta-analysis of uniportal versus multiportal videoassisted thoracoscopic lobectomy for lung cancer. Ann Cardiothorac Surg 2016;5:76-84.

3. Sihoe AD. Reasons not to perform uniportal VATS lobectomy. J Thorac Dis 2016;8:S333-43.

4. Pischik VG. Technical difficulties and extending the indications for VATS lobectomy. J Thorac Dis 2014;6:S623-30.

5. Chen YY, Lin TH, Tseng YL, et al. The video of uniportal VATS LUL lobectomy with stage bronchial closure. Asvide 2017;4:445. Available online: http://www.asvide. com/articles/1764

6. Chen YY, Lin TH, Tseng YL, et al. The video of uniportal VATS LUL lobectomy with stage bronchial closure when bronchial wall was torn. Asvide 2017;4:446. Available online: http://www.asvide.com/articles/1765

7. Gonzalez-Rivas D, Fieira E, Delgado M, et al. Is uniportal thoracoscopic surgery a feasible approach for advanced stages of non-small cell lung cancer? J Thorac Dis 2014;6:641-8.

8. Park JS, Kim HK, Choi YS, et al. Unplanned conversion to thoracotomy during video-assisted thoracic surgery lobectomy does not compromise the surgical outcome. World J Surg 2011;35:590-5.

9. Samson P, Guitron J, Reed MF, et al. Predictors of conversion to thoracotomy for video-assisted thoracoscopic lobectomy: a retrospective analysis and the influence of computed tomography-based calcification assessment. J Thorac Cardiovasc Surg 2013;145:1512-8.

10. Mallens WM, Nijhuis-Heddes JM, Bakker W. Calcified lymph node metastases in bronchioloalveolar carcinoma. Radiology 1986;161:103-4.

11. Wang GS, Wang J, Rao ZP, et al. Uniportal complete video-assisted thoracoscopic surgery lobectomy with partial pulmonary arterioplasty for lung cancer with calcified lymph node. J Thorac Dis 2015;7:2366-70.

doi: 10.21037/jovs.2017.08.16

Cite this article as: Chen YY, Lin TH, Chang CC, Huang WL, Yen YT, Tseng YL. Staged bronchial closure in uniportal video-assisted thoracoscopic anatomical resection for lung cancer with calcified lymph nodes. J Vis Surg 2017;3:149. 